\title{
Rectal Carriage of Carbapenemase Producing Enterobacteriaceae in Intensive Care Units of a Tertiary Care Hospital
}

\author{
Vignesh Kanna Balaji, K. Sudha*, B. Usha, Jeyakumari Duraipandian, \\ Joshy M. Easow and Swapna Muthuswamy
}

Annapoorna Medical College and hospital, Salem, Tamilnadu, India

*Corresponding author

\begin{tabular}{|c|}
\hline Keywords \\
\hline $\begin{array}{l}\text { Rectal carriage, } \\
\text { CarbaNP, Modified } \\
\text { carbapenemase } \\
\text { inactivation } \\
\text { method, } \\
\text { Carbapenemase- } \\
\text { producing } \\
\text { enterobacteriaceae }\end{array}$ \\
\hline Article Info \\
\hline $\begin{array}{l}\text { Accepted: } \\
17 \text { May } 2019 \\
\text { Available Online: } \\
\text { 10 June } 2019\end{array}$ \\
\hline
\end{tabular}

\section{Introduction}

Antibiotic resistance is observed in both pathogenic bacteria and normal commensal flora ${ }^{1}$. Current strategy applied for monitoring antibiotic resistance is through examination of pathogenic organisms and only periodic cross-sectional evaluation of resistance is undertaken for commensal flora. Members of family Enterobacteriaceae heavily colonize human gut and are the most frequent cause of bacterial infections in patients of all ages ${ }^{2}$. Humans are regularly exposed to new strains with novel genetic repertoires including antibiotic resistance through food, water, from other animate and inanimate sources in the community and hospital settings ${ }^{2}$.

Resistance amongst the commensal flora is a serious threat because, the highly populated 
ecosystem like the gut, may at a later stage, be the source of extra intestinal infections. Resistant strains may spread to other hosts or transfer resistance elements to other members of micro-biota including pathogens ${ }^{5}$. Extended spectrum Beta lactamase (ESBL) producing Enterobacteriaceae (ESBL-E) are rampantly reported in India and carbapenems are the drug of choice ${ }^{3}$ for ESBL-E. Gastrointestinal tract may serve as a reservoir for Carbapenem Resistant Enterobacteriaceae (CRE), resulting in cross transmission within the health care setting ${ }^{4}$.

Infection caused by ESBL-E is associated with antibiotic abuse or lengthy hospital stay and poor compliance with infection control measures. ESBL-E infections are also linked to increased mortality, largely due to lack/delay in effective therapy. The increasing use of carbapenems for empirical therapy of hospital-acquired sepsis has led to a rapid global dissemination of CarbapenemaseProducing Enterobacteriaceae (CPE) ${ }^{5}$.

Patients of medical units with high levels of antibiotic consumption are also prone for colonization of Multi drug resistant pathogens includes ESBL and Carbapenemase producers. Intestinal carriage serves as a reservoir of $\mathrm{CPE}$ and can promote crosstransmission in health care settings. Awareness of the fecal carriage of ESBL and CPE bacteria is very important for the clinicians, microbiologists, infection control practitioners and epidemiologists. Global data shows that the prevalence of these bacteria poses serious threat to people in both community as well as hospital setting ${ }^{6}$.

Study for the detection of Carbapenemase producing Enterobacteriaceae is pertinent for framing antibiotic and infection control policies. Hence, we intended to screen the $\mathrm{CPE}$ in fecal sample of ICU patients.

\section{Materials and Methods}

The prospective cohort study was conducted in the department of Microbiology. Institutional Ethical clearance was obtained and the samples were collected from April 2017 to September 2017. A total of 40 rectal swabs were obtained from ICU patients after getting informed consent from the patients or their attenders. All the ICU patients who stayed for more than 3days were included in the study. Exclusion criteria include previously hospitalized patients, elimination of other than Enterobacteriaceae organisms.

Rectal swabs were inoculated onto MacConkey agar with $5 \mathrm{mg} / \mathrm{l}$ of cefotaxime and incubated at $37^{\circ} \mathrm{C}$ for $24 \mathrm{hrs}$. Identification was made by following standard microbiological methods used for identification of Enterobacteriaceae.

\section{Antimicrobial susceptibility testing}

On Muller-Hinton agar, lawn culture of test organism was made and antibiotic disc were placed on it amikacin $(30 \mu \mathrm{g})$, gentamicin $(30 \mu \mathrm{g}), \quad$ cefotaxime $(30 \mu \mathrm{g})$, ceftazidime $(30 \mu \mathrm{g})$, ceftriaxzone $(30 \mu \mathrm{g})$, ciprofloxacin $(5 \mu \mathrm{g})$, meropenem $(10 \mu \mathrm{g})$, imipenem $(10 \mu \mathrm{g})$, co-trimoxazole $\quad(1.25 / 23.75 \mu \mathrm{g}), \quad$ and piperacillin/tazobactam $(100 / 10 \mu \mathrm{g}) \quad$ and incubated at $37^{\circ} \mathrm{C}$ for $24 \mathrm{hrs}$; interpretation was based on CLSI guidelines 2017.

\section{Carbapenemase detection}

\section{RAPIDEC ${ }^{\circledR}$ Carba NP}

Carbapenemase producing Enterobacteriaceae was detected by using BioMerieux RAPIDEC ${ }^{\circledR}$ Carba NP kit. The tests were performed according to the manufacturers recommendation ${ }^{7}$. A loop of the bacterial colony was picked up from overnightincubated Muller-Hinton agar plate and mixed 
into API suspension medium (provided with kit); the bacterial suspension was then transferred to wells in the test strip and incubated at $37^{\circ} \mathrm{C}$. Visual readings of the test strip was done after $30 \mathrm{~min}$ and also after 2 hrs. A positive test corresponded to a color change from red to yellow- orange, whereas a red color indicated a negative result.

\section{mCIM (Modified Carbapenemase inactivation method) ${ }^{8}$}

Isolate was emulsified in $2 \mathrm{ml}$ of Trypticase soy broth and $10 \mu \mathrm{g}$ meropenem disk was added to each tube, incubated at $35^{\circ} \mathrm{C}$ in ambient air for 4 hrs.

E. coli ATCC 25922 was inoculated as lawn culture onto Muller Hinton agar medium, and the incubated meropenem disk was placed on the MHA, after which the MHA was incubated at $35^{\circ} \mathrm{C}$ for $18-24 \mathrm{hrs}$.

The zone of inhibition was measured and reported as positive if zone diameter is 6$15 \mathrm{~mm}$, and negative if $>19 \mathrm{~mm}$. If the test isolate produces a Carbapenemase, the meropenem in the disk will be hydrolysed and there will be no inhibition or limited growth inhibition of the meropenem susceptible E.coli ATCC 25922.

\section{Results and Discussion}

A total of 25 isolates were obtained from rectal swabs of 40 ICU patients. Out of 25 isolates, ten were $(10 / 25,22 \%) E$. coli and fifteen were $(15 / 25,42 \%)$ Klebsiella species. All the isolates were evaluated for antimicrobial susceptibility to various antimicrobials. Maximum sensitivity was found against piperacillin/tazobactam (61.53\%). All the isolates (100\%) were resistant to cotrimoxazole, imipenem and meropenem. whereas $60-90 \%$ isolates were highly resistant to the other antimicrobials tested.

All the isolates were subjected for the detection of Carbapenemase production by BioMerieux RAPIDEC $^{\circledR}$ CarbaNP kit, a colorimetric assay. Out of 25 isolates 20 were positive for Carbapenemase production out of which, seven were $(7 / 20,36 \%)$ E.coli and thirteen were $(13 / 20,64 \%) \quad$ Klebsiella species.

Modified Carbapenemase inactivation test is performed for all the isolates. Out of 25 isolates, 23 were positive. Eight were (8/23, $34 \%)$ E.coli and fifteen were $(15 / 23,66 \%)$ Klebsiella species. In CarbaNP test and mCIM, 5 and 2 isolates were negative respectively (Fig. 1 and 2; Table 1).

Table.1 Comparison of CarbaNP and mCIM test

\begin{tabular}{|c|c|c|}
\hline Isolate & CarbaNP (20) & mCIM (23) \\
\hline E.coli & $7(36 \%)$ & $8(34 \%)$ \\
\hline Klebsiella spp & $13(64 \%)$ & $15(66 \%)$ \\
\hline
\end{tabular}


Figure.1 BioMerieux Rapidec CarbaNP test

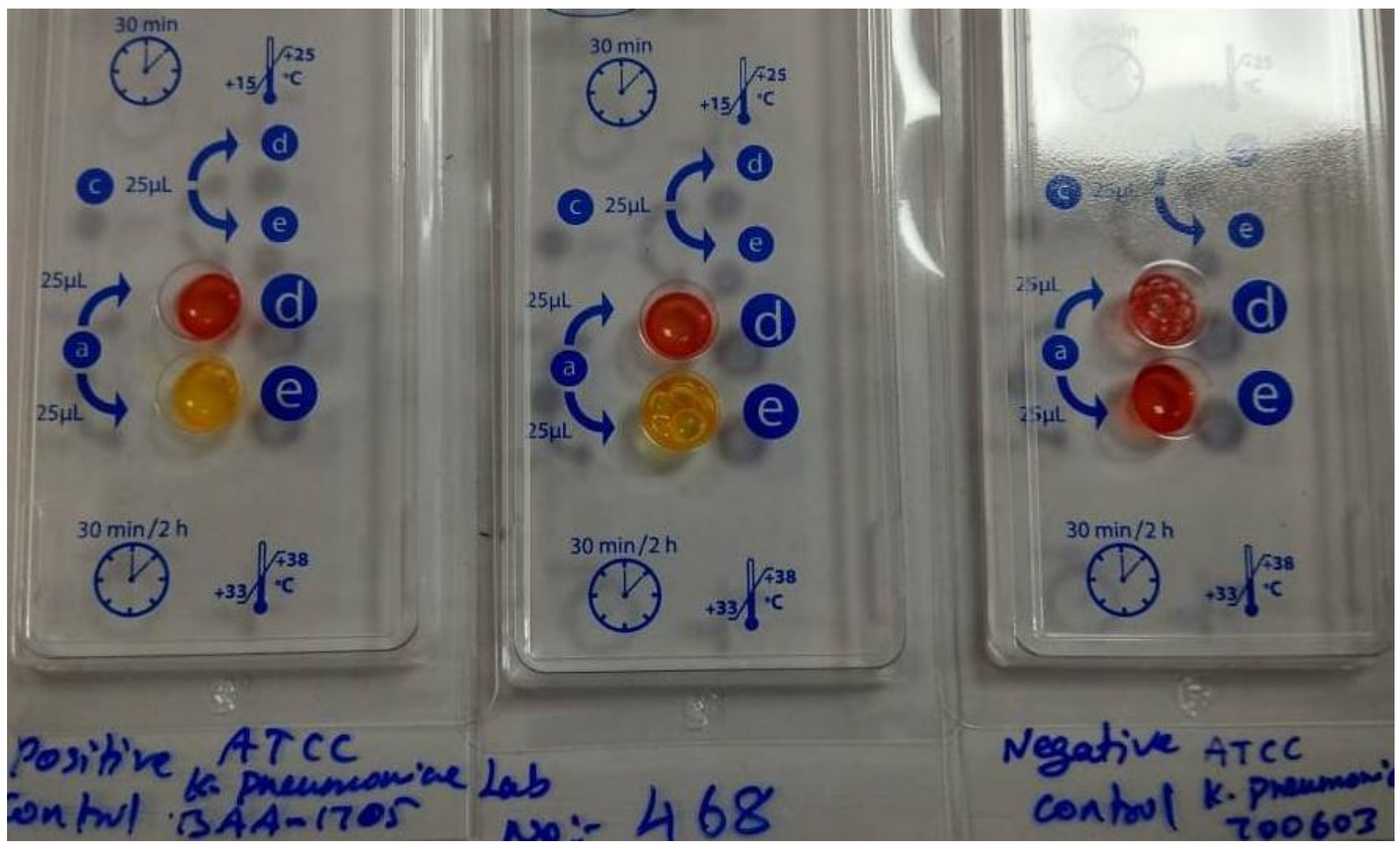

Figure.2 Modified carbapenemase inactivation method

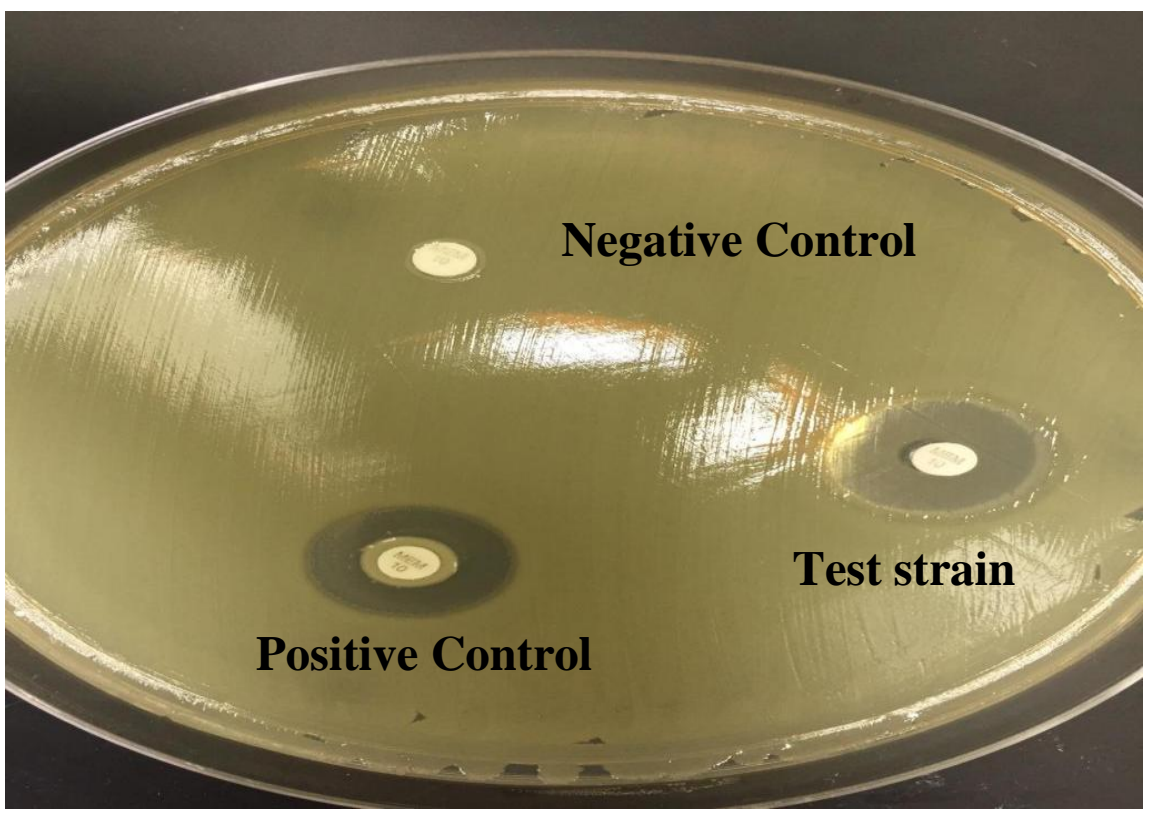


Carbapenems are the most effective antimicrobial agent against Gram-negative bacteria and have a broad spectrum of antibacterial activity against members of Enterobacteriaceae, Pseudomonas aeruginosa, Acinetobacter baumanni complex ${ }^{9}$. They are generally used as last-resort drugs for the treatment of infection caused by multidrug resistant bacteria. Mechanism of resistance to Carbapenems include production of Betalactamase, efflux pumps, and mutation that alter the expression and /or function of porins and penicillin-binding proteins9. The alarming increase of CRE prevalence worldwide is worrisome. In many endemic settings of UAE and Iran, prevalence as high as $24.7 \%-29.8 \%$ have been noted ${ }^{10}$. Mohan $e t$ $a l^{11}$ in 2017, documented the prevalence rate of CRE as $18.7 \%$ in India. The faecal carriage of CRE acts as a reservoir for dissemination of these multidrug-resistant pathogens through cross-transmission. These highly drug-resistant organisms may stay for prolonged periods in the intestinal tract without causing any infections or may serve as a source of endogenous urinary tract infections, intra-abdominal infections or even translocate through the gut epithelium to cause sepsis $^{12}$.

Preventing the transmission of CP-CRE is a high priority for all the institutions. Screening the contacts of CP-CRE infected patients is essential to curb transmissions and control outbreaks. However, approaches to routine screening for CRE carriage vary depending on institutional epidemiology, resources and policies $^{5}$. Baseline surveillance is recommended to determine the prevalence and types of Carbapenemase enzymes circulating in an institution ${ }^{7}$. Although molecular assays are expensive method for CP-CRE screening, the fast turnaround time, high sensitivity, specific genetic information, and availability of FDA-approved or CEmarked assays are appealing. Culture-based methods are yet to be approved, less sensitive than molecular methods, labour intensive and slow, but have a lower reagent $\operatorname{cost}^{7}$.

In our study $22 \%$ E.coli and $42 \%$ Klebsiella spp were resistant to carbapenems, which was similar to the results shown in the study conducted by Xu $e t a l^{10}$.

The Carba NP test has multiple benefits. It is inexpensive, rapid, reproducible, and highly sensitive and specific. It eliminates the need for using other techniques to identify carbapenemase producers that are timeconsuming and less sensitive or specific. Using this accurate test would improve detection of patients infected or colonized with Carbapenemase producers. A study conducted by Atul Garg et $a l^{13}$ in India, showed the sensitivity and specificity of BioMerieux RAPIDEC ${ }^{\circledR}$ CarbaNP test as $92.6 \%$ and $96.2 \%$ which is in concordance with our study. A study from the National Reference Center for Antibiotic Resistance, France, Dortetet $a l^{14}$ documented a sensitivity of $99 \%$ and specificity of $100 \%$. Poirel and Nordmann ${ }^{15}$ tested this kit with precharacterized strains and documented a sensitivity and specificity of $96 \%$. In this study the Carba NP test showed positive for 20 isolates of 25 in which, seven were (7/20, $36 \%)$ E. coli and thirteen were $(13 / 20,64 \%)$ Klebsiella species.

Clinical and Laboratory Standards Institute (CLSI), 2017 included the "modified CIM (mCIM)" as a Carbapenemase screening test for Enterobacteriaceae (CSLI M100-S27) ${ }^{8}$ which is relatively simple with the sensitivity of $>99 \%$ and specificity of $>99 \%$ for detection of CPE. Our study showed 23 isolates positive out of 25 by Modified Carbapenemase inactivation test. In this eight were $(8 / 23,34 \%) \quad$ E.coli and fifteen were (15/23, 66\%) Klebsiella species. In CarbaNP test and $\mathrm{mCIM}, 5$ and 2 isolates were negative 
respectively. These results concordance with the study conducted by Goel $\mathrm{N}$ et al. Wherein, organisms producing low Carbapenemase activity enzymes, thickness of the inoculum and the disc potency are likely to influence the test results of mCIM with an overnight delay ${ }^{6}$.

\section{Limitation}

A major drawback of our current study is that it was performed as a pilot study over a short duration. Due to time and resource constrains, molecular characterization and other nonenzymatic mechanisms mediating carbapenem resistance, such as upregulated efflux pumps, porin defects, and hyper production of AmpC beta-lactamase were not performed in this study.

Hence concluded that, overall both the methods showed similar results. The MCIM is relatively simple and reliable, but requires good technical skill compared to RAPIDEC ${ }^{\circledR}$ CarbaNP. Early screening is needed for prevention of transmission in hospitalised patients. Surveillance studies might help in the implementation of infection control measures to curtail the spread of these isolates, both in the hospital and in the community.

\section{Acknowledgement}

We would like to express my gratitude to late Dr Lavina Lilly Francis in her absence, who came up with the idea of this research topic.

\section{References}

1. Andremont A. Commensal flora may play key role in spreading antibiotic resistance. ASM News. 2003; 69(12):601-7.

2. Grundmann H, Livermore DM, Giske CG, Canton R, Rossolini GM, Campos J et al. Carbapenem-non-susceptible Enterobacteriaceae in Europe: conclusions from a meeting of national experts. Euro Surveil. 2010;15:46-50.

3. Kothari C, Gaind R, Singh LC, Chellani H, Saxena S, Deb $\mathrm{M}$ et al. Community acquisition of beta-lactamase producing Enterobacteriaceae in neonatal gut. BMC Microbiol.2013; 13:136-8.

4. Walsh TR, Weeks J, Livermore DM, Toleman MA. Dissemination of NDM-1 positive bacteria in the New Delhi environment and its implication for human health: An environmental point prevalence study. Lancet Infect Dis. 2011; 11:355-62.

5. Mikael A, Astrid L, Dominique D, Dimitri M, Naike B, Claire P. Extendedspectrum beta-lactamase producing Enterobacteriaceae in the intensive care unit: acquisition does not mean crosstransmission. BMC Infect Dis 2016; 16:147-9.

6. Goel N, Aggarwal R, Chaudhary U, Rohilla $\mathrm{H}$, Dahiya S. Fecal carriage of Extended spectrum beta lactamase producing organisms in a teaching tertiary care hospital of North India. Intl J Med Dent sci $2014 ; 3(1): 246-9$.

7. Gajanand M, Rajni G, Deepak K, Gaurav $\mathrm{K}$, Kunj BG, Verma PK et al. Risk factors for fecal carriage of Carbapenemase producing Enterobacteriaceae among intensive care unit patients from a tertiary care centre in India. BMC Microbiol 2016; 16:138-40.

8. Clinical and Laboratory Standards Institute. Performance standards for Antimicrobial susceptibility testing; Twenty-seventh information supplement. CLSI document M100S27. Wayne PA: Clinical and Laboratory Standards Institute January 2017.

9. Pragasam AK, Veeraraghavan B, 
Bakthavatchalam YD, Gopi R, Aslam RF. Strengths and limitations of various screening methods for carbapenemresistant Enterobacteriaceae including new method recommended by clinical and laboratory standards institute, 2017: A tertiary care experience. Indian J Med Microbiol 2017; 35:116-9.

10. Xu Y, Gu B, Huang M, Liu H, Xu T, Xia $\mathrm{W}$, et al. Epidemiology of carbapenem resistant Enterobacteriaceae (CRE) during 2000-2012 in Asia. J Thorac Dis 2015; 7:376-85.

11. Mohan B, Prasad A, Kaur H, Hallur V, Gautam N, Taneja N. Fecal carriage of carbapenem-resistant Enterobacteriaceae and risk factor analysis in hospitalised patients: A single centre study from India. Indian J Med Microbiol 2017; 35:555-62.
12. Carlet J. The gut is the epicentre of antibiotic resistance. Antimicrob Resist Infect Control 2012; 1:39.

13. Garg A, Garg J, Upadhyay GC, Agarwal A, Bhattacharjee A. Evaluation of the Rapidec Carba NP test kit for detection of carbapenemase-producing Gramnegative bacteria. Antimicrobial agents and Chemotherapy. 2015; 59(12):78702.

14. Dortet L, Cuzon G, Nordmann P. Dissemination of carbapenemaseproducing Enterobacteriaceae in France, 2012. J Antimicrob Chem. 2013; 69(3): 623-7.

15. Nordmann P, Poirel L, Dortet L. Rapid detection of carbapenemase-producing Enterobacteriaceae. Emerging Infect Dis. 2012; 18(9): 1503.

\section{How to cite this article:}

Vignesh Kanna Balaji, K. Sudha, B. Usha, Jeyakumari Duraipandian, Joshy M. Easow and Swapna Muthuswamy. 2019. Rectal Carriage of Carbapenemase Producing Enterobacteriaceae in Intensive Care Units of a Tertiary Care Hospital. Int.J.Curr.Microbiol.App.Sci. 8(06): 22172223. doi: https://doi.org/10.20546/ijcmas.2019.806.264 